\title{
Immunohistochemical analysis of spinal cord components in mouse model of experimental autoimmune encephalomyelitis
}

\author{
Grazyna Pyka-Fosciak ${ }^{1}$, Mariusz Stasiolek ${ }^{2}$, Jan A. Litwin ${ }^{1}$ \\ ${ }^{1}$ Department of Histology, Jagiellonian University Medical College, Krakow, Poland \\ ${ }^{2}$ Department of Neurology, Medical University of Lodz, Lodz, Poland
}

\begin{abstract}
Introduction. Experimental autoimmune encephalomyelitis (EAE) is a widely used animal model for studying immunopathology of multiple sclerosis (MS) because it repeats the hallmarks of the human disease, such as focal inflammation and demyelination of the central nervous system, subsequently leading to axonal and neuronal loss. The interrelationships, timing and sequence of different pathological processes that lead to histologically observed lesions in SM are still incompletely understood.

Material and methods. EAE was induced in female C57Bl/6 mice by active immunization with MOG35-55 antigen. Development of the neurological symptoms in the animals was monitored and on that basis spinal cords were collected in three successive phases of the disease (onset, peak, chronic). Total leukocytes, T cells, macrophages/ /microglia, oligodendrocytes, damaged axons and surviving neuronal cell bodies were visualized using appropriate immunohistochemical markers and their density was quantitatively assessed using image analysis software. Results. The density of all studied cells except neurons was significantly higher in EAE mice than in the control mice. The density of total leukocytes, T cells, and damaged axons increased from the onset to the peak phase and decreased in the chronic phase to reach values lower than those in the peak phase. The density of macrophages/ /microglia increased in the peak phase and remained at the elevated level in the chronic phase. Oligodendrocytes showed the highest density in the onset phase and gradually decreased afterwards. The density of neuronal cell bodies decreased only in the chronic phase of the disease.

Conclusions. In mouse model of EAE, inflammatory cells predominate in the early phases of the disease. This study shows for the first time that inflammation precedes oligodendrocyte death and neuronal loss and that macrophages/ microglia are the only cells persisting in large numbers in the chronic phase of the disease, probably because of the switch from proinflammatory to anti-inflammatory phenotype. (Folia Histochemica et Cytobiologica 2018, Vol. 56, No. 3, 151-158)
\end{abstract}

Key words: EAE; spinal cord; leukocytes; T cells; macrophages; microglia; oligodendrocytes; neuronal damage; immunohistochemistry

\section{Introduction}

Experimental autoimmune encephalomyelitis (EAE) is a widely used animal model for studying immunopathology of multiple sclerosis (MS) because it

Correspondence address: Grazyna Pyka-Fosciak

Department of Histology, Jagiellonian University

Medical College,

Kopernika 7, 31-034 Krakow, Poland

e-mail: gpfosciak@cm-uj.krakow.pl repeats the hallmarks of the human disease, such as focal inflammation and demyelination of the central nervous system (CNS), subsequently leading to axonal and neuronal loss [1]. In C57Bl/6 mice, EAE can be induced by active immunization with MOG35-55 antigen which triggers the inflammatory demyelinating disease [2]. The clinical symptoms in EAE reflect inflammation localized predominantly in the spinal cord, although MOG-induced EAE also targets the optic nerve [3]. The neuroinflammatory response is manifested by infiltrations of $\mathrm{T}$ cells, $\mathrm{B}$ cells and 


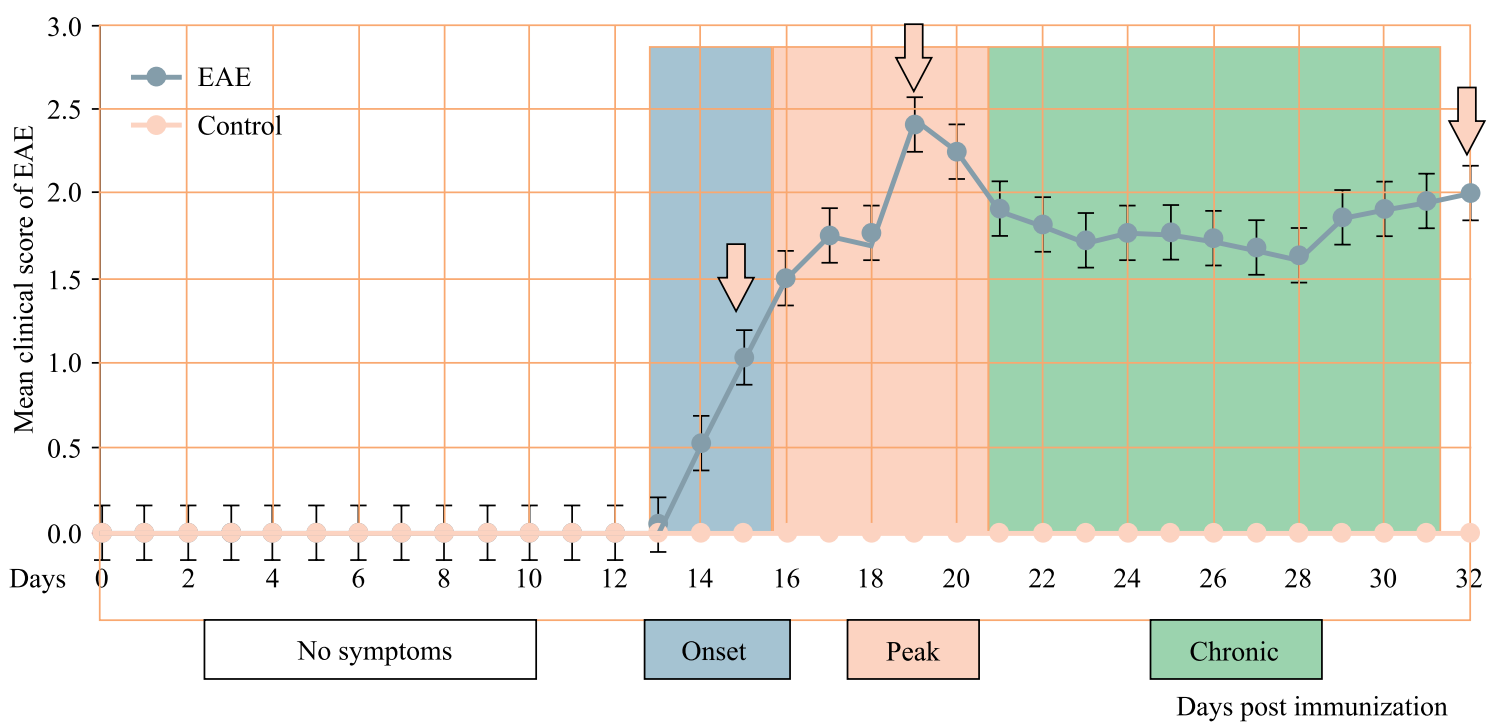

Figure 1. Development of neurological symptoms in experimental autoimmune encephalitis (EAE) mice. EAE was elicited and mice were scored for EAE symptoms over 30 days using the scale as described in Methods. Day 0 was the day of immunization. Arrows show three time points (tissue collection for EAE and control groups) representing three successive phases of the disease. Values represent means \pm SEM.

macrophages, as well as by formation of focal demyelination plaques in the CNS, similar to the pathology seen in MS [4]. The interrelationships, timing and sequence of different pathological processes that lead to histologically observed lesions in MS are still incompletely understood. It is also unclear, whether neuroinflammation or demyelination are primary or secondary events during development of the disease [5]. Hence, EAE is a good model for studying MS mechanisms and for testing or developing drugs [6].

The aim of the present study was to investigate the progressive neurodegenerative process in EAE mice by analyzing the occurrence and number of the demyelination plaque components: leukocytes, macrophages/microglial cells, oligodendrocytes, as well as damaged axons and surviving nerve cells at different stages of the disease. These parameters reflect spinal cord inflammation and neurodegeneration in EAE which occur in both white and gray matter.

\section{Material and methods}

Animals. Female C57Bl/6 mice (18 animals for EAE and 18 for control) aged 10-11 weeks were habituated for at least 1 week before the experiment and then were immunized. The animals were housed under controlled environmental conditions (LD 12:12 h regime in air-conditioned rooms: $22 \pm 2^{\circ} \mathrm{C}$, $55 \pm 10 \%$ humidity) and allowed free access to food and water throughout the experiment. All experiments were conducted in compliance with the Council Directive 2010/63EU of the European Parliament and the Council of 22 September 2010 on the protection of animals used for scientific purposes and approved by the First Local Ethics Committee of the Jagiellonian University Medical College, Krakow, Poland.

Induction of EAE. For induction of EAE, Hooke Kits ${ }^{\mathrm{TM}}$ EAE Emulsion (Hooke Laboratiores, Lawrence, Massachusetts USA) were used. Kits contained MOG35-55 antigen in an emulsion with Complete Freund's Adjuvant (CFA) including Mycobacterium tuberculosis (H37Ra). Emulsion was administered subcutaneously at two sites $(100 \mu$ l between the ears and $100 \mu \mathrm{l}$ in hind flank). Each mouse was also injected intraperitoneally (i.p.) with $340 \mu \mathrm{l}$ of Bordatella pertussis pertussis toxin (PTx) dissolved in phosphate-buffered saline (PBS) (Hooke Laboratiores) $2 \mathrm{~h}$ after the administration of the emulsion and again $24 \mathrm{~h}$ later. PTx enhanced EAE development by providing additional adjuvant and facilitating entrance of autoimmune T cells into the CNS [2]. Control mice were injected with CFA and PTx only (Hooke Control Kits ${ }^{\mathrm{TM}}$, Hooke Laboratiores, USA) according to the same schedule.

Monitoring of the course of the disease and study design. All mice were weighed and examined daily for the neurological symptoms of EAE, scored according to the following scale: (0) no symptoms; (1) floppy tail; (2) hindlimb weakness; (3) hindlimb paralysis; (4) forelimb paralysis; (5) mouse moribund or dead ( 0.5 gradations represent intermediate scores) [7].

For histological analysis EAE mice were sacrificed at three different time points representing three phases of disease: onset phase (day 15; $\mathrm{n}=6$ ), peak phase (day 19; $\mathrm{n}=6$ ) and chronic phase (day 32; $\mathrm{n}=6$ ) (Fig. 1). Control 
mice were sacrificed at the same time points $(n=6$ for each phase).

All EAE mice showed initial symptoms of the disease between 13th and 16th day after immunization and this was the onset phase in our experiment. The peak of disease almost always occurred 3-4 days later, between days 16 and 21. The maximum scores of the peak phase continued for several days and then mice partially recovered (chronic phase). During the progress of EAE, changes in body weight also reflected disease severity. Mice steadily increased their body weight until disease onset. Afterwards, mice gradually lost $5-10 \%$ of their weight and after the peak of disease they slowly gained weight again.

Tissue collection and processing. Animals were anaesthetized i.p. with $100 \mathrm{mg} / \mathrm{kg}$ ketamine and $10 \mathrm{mg} / \mathrm{kg}$ xylazine and transcardially perfused with ice-cold PBS for $10 \mathrm{~min}$, followed by $4 \%$ paraformaldehyde for the next $10 \mathrm{~min}$. Spinal cords were carefully removed from the vertebral canal and postfixed in the same fixative for $4 \mathrm{~h}$. After overnight incubation in $5 \%$ sucrose at $4^{\circ} \mathrm{C}$, tissue was embedded in OCT (Shandon Cryomatrix, Thermo Fisher Scientific, Rockford, IL USA) and snap-frozen at $-80^{\circ} \mathrm{C}$. The examined area of the spinal cord included the lumbar part, a region commonly and rapidly affected in EAE. Serial cryosections $10 \mu \mathrm{m}$ - thick were cut at $100 \mu \mathrm{m}$ intervals, collected on poly-L-lysine coated slides and air dried.

Immunofluorescence staining. Immunohistochemistry was performed using the following primary antibodies: rat anti-CD45 (1:100, Thermo Fisher Scientific, MA181247) for total leukocytes, rat anti-CD3 (1:100, Thermo Fisher Scientific, cat. \# MA1-80783) for T cells, rabbit anti-ionized calcium binding adaptor molecule (anti-Iba1) for activated macrophages and microglial cells (1:200, Synaptic Systems, Goettingen, Germany, cat. \# 234003), rabbit anti-2',3'-Cyclic nucleotide 3'-phosphodiesterase 1 (anti-CNP1) for oligodendrocytes (1:500, Synaptic Systems, cat. \# 355002), rabbit anti-beta-amyloid precursor protein (beta-APP) for detection of injured axons (1:100, Synaptic Systems, cat. \# 218903) and rabbit anti-NeuN for detection of surviving neurons (1:100, Thermo Fisher Scientific, cat. \# PA5-37407).

In short, the sections were preincubated for $40 \mathrm{~min}$ in PBS containing 5\% normal goat serum (Sigma-Aldrich, St. Louis, MO, USA), $0.01 \%$ sodium azide, $0.05 \%$ thimerosal, $0.1 \%$ bovine serum albumin, $0.5 \%$ Triton $\mathrm{X}-100$, and $2 \%$ dry milk. They were next incubated overnight at room temperature with primary antibodies and after a rinse in PBS incubated for 90 min with the secondary goat anti-rat Alexa488-conjugated antibodies (1:100, Jackson IR, West Grove, PA, cat. \# 112-545-167), goat anti-rabbit Alexa488-conjugated antibodies (1:100, Jackson IR, West Grove, PA, cat. \# 111-545-144) or Cy3-conjugated goat anti-rat antiserum (1:300, Jackson IR, West Grove, PA, 112-165-167). Sections were washed three times in PBS and mounted in glycerol/PBS solution.

Microscopy and morphometry. The sections were examined under Olympus BX50 brightfield/epifluorescence microscope (Olympus, Tokyo, Japan). Images of the spinal cords were recorded using Olympus DP71 digital CCD camera, stored as TIFF files and processed for quantitative analysis using Cellsense Dimension Software (Olympus). A total of at least 24 slides were analyzed per experimental group $(n=6)$. The density of the immunopositive cells was assessed in sections encompassing the whole area occupied by the gray and white matter and expressed in two ways: as the number of T cells and surviving neurons and as the immunoreactive profile area in case of total leukocytes, macrophages, oligodendrocytes and damaged axons. The analysis was done blind (the observer did not know whether the specimen comes from the control or EAE mouse).

Statistical analysis. The obtained results were analyzed by the Mann-Whitney U-test for nonparametric data using Prism 5.0 software (GraphPad, La Jolla, California, USA). $\mathrm{P}<0.05$ was regarded as statistically significant.

\section{Results}

All mice immunized with MOG developed neurological symptoms of EAE. Control mice did not develop any signs of neurological disease.

\section{Histological examination of spinal cords of EAE and control mice}

Immunoreactivity for CD45 showed large accumulations of leukocytes in the white and gray matter in EAE mice and much less leukocytes in control mice. In all phases of the disease, leukocytes formed local aggregates with very high cell density, indicative of the presence of inflammatory foci.

The immunostaining of the spinal cord sections with anti-CD3 antibody revealed areas containing numerous CD3-positive $\mathrm{T}$ cells in white matter of EAE mice and relatively few in gray matter of EAE and control mice.

Iba1-positive activated macrophages/microglial cells showed an equal distribution in white and gray matter and were much more numerous in EAE mice, as compared to control animals.

Oligodendrocytes revealed by CNP1 immunoreactivity were abundant, mainly in the white matter of both EAE and control mice. In EAE mice they seemed to be most numerous during the onset phase and to decrease in number in the following phases of the experimental disease. 
Axonal damage was observed in the white matter of EAE mice in all phases of the disease with the highest immunoreactivity at the peak of neurological symptoms. Control mice did not show immunostaining indicative of axonal damage.

NeuN-positive cells bodies of surviving neurons in gray matter seemed to be less numerous in EAE mice than in control mice only in the chronic phase of EAE.

\section{Quantitative analysis of spinal cord sections of EAE and control mice}

The density of all studied cells was significantly higher in EAE mice than in the control mice. The only exception were neurons - the density of neuronal cell bodies in EAE mice was comparable to that in control mice in the onset and peak phases. The density of total leukocytes, $T$ cells, and damaged axons generally revealed a similar phase-dependent course in EAE mice: it increased from the onset to the peak phase and decreased in the chronic phase (Figs. 2, 3,6) to reach values lower than those in the peak phase. The density of activated macrophages/microglia increased in the peak phase and remained at the elevated level in the chronic phase (Fig. 4). Oligodendrocytes showed the highest density in the onset phase and gradually decreased afterwards. In the chronic phase, their density was even significantly lower than that in the control mice (Fig. 5). The number of neuronal cell bodies decreased only in the chronic phase and there were no significant differences between the other phases of the disease (Fig. 7). Numerical results of the measurements are presented in Table 1.

\section{Discussion}

Results of this study show dynamics of cells involved in the pathogenesis of EAE and progress of the main histopathological changes, axonal damage and neuronal death during development and progression of the disease. The investigated parameters were correlated with three successive phases of EAE: onset, peak and chronic, characterized by neurological symptoms [2]. It could be expected that cells involved in the inflammatory process in the onset phase would reveal higher density as compared with the control, their density would further increase in the peak phase and decline during the chronic phase, as suggested by partial recovery of the mice in that phase.

Indeed, the density of T cells, the key players in the pathology of EAE and SM follows such pattern. It is widely accepted that EAE is initiated by T helper (Th) cells which escape self-tolerance mechanisms and bind myelin proteins via their TCR receptors. In further phases of the disease, two subsets of T cells:
IFN $\gamma$-secreting Th1 cells and IL-17-secreting Th17 cells, acting in cooperation with antigen presenting cells, recruit effector cells of EAE: monocytes/macrophages and microglia to the areas of the developing inflammation (mostly perivascular regions of the central nervous system and meninges) and the phagocytotic activity of the latter cells is directly responsible for demyelination $[8,9]$. A decrease in the number of T cells at later stages of EAE was also reported by Herrero-Herranz et al. [10].

Total leukocytes showed a similar behavior. CD45 antibody detects T cell, B cells, neutrophils and monocytes/macrophages. B cells and neutrophils have been recently demonstrated to play significant roles in the development and progression of EAE. B cells amplify the autoimmune process by taking up myelin antigens and presenting them to Th cells, they also produce anti-myelin antibodies [11, 12]. It has been recently demonstrated that some, not yet identified, secretory products of B cells obtained from MS patients induce apoptosis of oligodendrocytes and neurons in vitro [13].

Neutrophils, which were found to infiltrate EAE lesions in large quantities, together with lymphocytes and macrophages [14], seem to contribute to the pathogenesis of EAE via several mechanisms. They significantly participate in the disruption of blood-brain/spinal cord barrier, thus facilitating transmigration of other cells involved in EAE [15]. By producing IL- $1 \beta$, neutrophils promote differentiation of a Th cell subset important for the development of EAE [16], they also secrete cofactors necessary for maturation of professional antigen presenting cells in EAE lesions [17].

We observed that the density of macrophages/ /microglia increases during the first two phases of EAE and remains at the elevated level in the chronic phase. This behavior well corresponds with the activity of these cells during progression of the disease. The initial phase of EAE is characterized by massive infiltration of the nerve tissue by blood monocytes which are transformed into macrophages and by activation of resident microglia [18]. The vast majority of these cells show "classically activated" proinflammatory M1 phenotype [19] and they secrete cytokines promoting tissue damage, neuronal death and demyelination, the latter being additionally intensified by the phagocytotic activity of both cell types. This involvement of M1 macrophages/microglia greatly contributes to the development of the lesions in the initial and peak phases [20]. However, later in the course of EAE, M1 phenotype is gradually replaced by M2 macrophages/ /microglia [21] showing anti-inflammatory properties promoting tissue repair and subsequent functional 


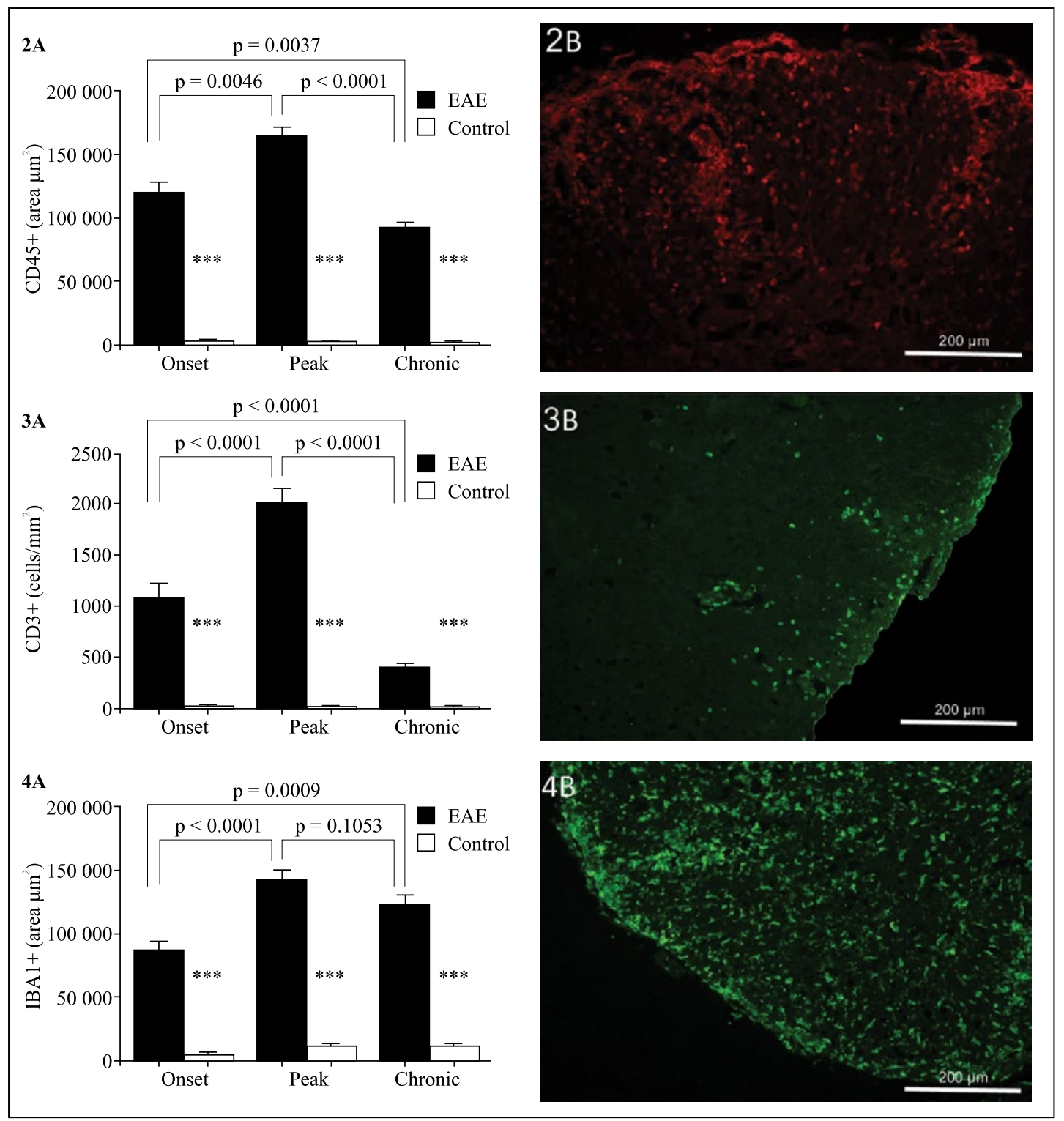

Figures 2-4. Cell density in spinal cords of EAE mice in three successive phases of the disease (A) with representative micrographs showing immunostained cells in the peak phase of EAE (B). Fig. 2. Total leukocytes immunostained for CD45. Fig. 3. T cells immunostained for CD3. Fig. 4. Macrophages/microglia immunostained for IbA1. Histomorphometry was performed as described in Methods. Values represent means \pm SEM; $\mathrm{n}=6$ per group; EAE $v s$ control: $* * * \mathrm{p}<0.0001$.

improvement observed in the chronic phase of EAE. This seems to explain our finding of the retention of macrophages/microglia in EAE lesion after the peak phase of the disease.

It has been a striking observation made in this study that already at the onset phase of EAE the number of oligodendrocytes is substantially higher than in the control mice. Under demyelinating conditions, oligodendrocyte precursor cells (OPS) are recruited to the lesion site and maturate into myelinating oligodendrocytes [22] involved in tissue repair. Our observation indicates that the recruitment of OPC and their maturation occurs very early, shortly after the beginning of the demyelination processes. High density of oligodendrocytes persists in the peak phase; however, intense demyelination and extensive accumulation of myelin debris in the lesion results in the inhibition of OPC maturation [23] and inefficient 


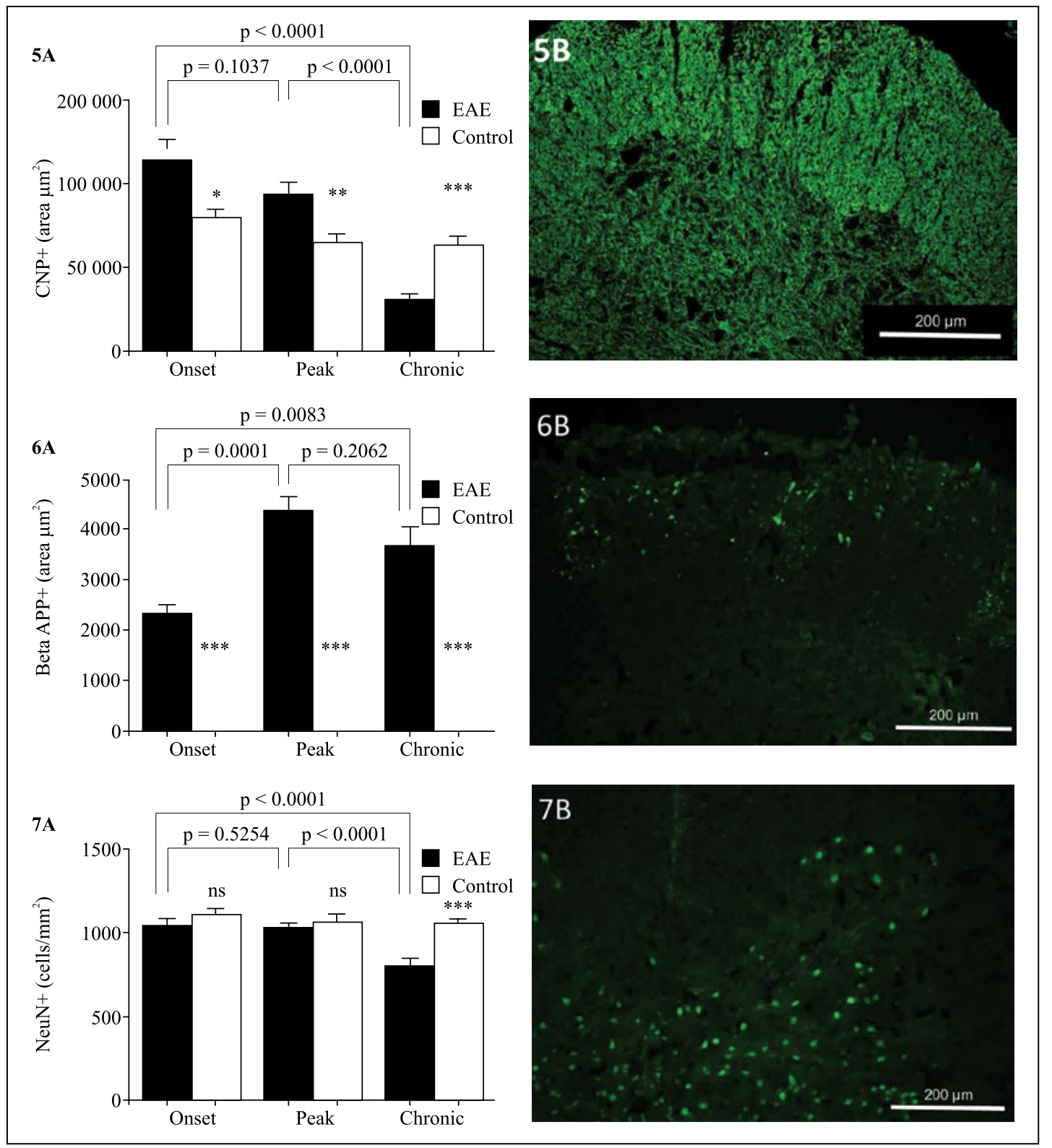

Figures 5-7. Cell and axonal damage density in spinal cords of EAE mice in three successive phases of the disease (A) with representative micrographs showing immunostained structures in the peak phase of EAE (B). Fig. 5. Oligodendrocytes immunostained for CNP1 (note much higher density in white matter, top). Fig. 6. Damaged axons immunostained for beta APP. Fig. 7. Surviving neurons immunostained for NeuN. Histomorphometry was performed as described in Methods. Values represent means \pm SEM.; $\mathrm{n}=6$ per group; EAE $v s$ control: ${ }^{*} \mathrm{p}<0.05,{ }^{*} \mathrm{p}<0.001,{ }^{* * *} \mathrm{p}<0.0001$.

re-myelination. The density of oligodendrocytes showed a decrease in the chronic phase, to a level even significantly lower than that in the control mice, indicating a substantial loss of these cells. Indeed, advanced EAE is characterized by a massive oligodendrocyte death [24]. The main mechanism of death seems to be caspase-mediated apoptosis [25]. It has been a matter of debate, whether oligodendrocyte death precedes local inflammation or whether inflammation induces death of these cells. Results of this study clearly point to the latter sequence of events, at least in the model of MOG-induced EAE, since a decrease in oligodendrocyte density was noted no sooner than in the chronic phase of EAE.

According to the expectations, axonal damage shows progress in the onset and peak phases of EAE and becomes stabilized in the chronic phase, in which the demyelination and remyelination processes become balanced. This result confirms earlier ultrastructural data indicating that axonal damage in EAE 
Table 1. Density of cells and damaged axons in the spinal cord of EAE mice in three phases of the disease

\begin{tabular}{|c|c|c|c|c|c|c|}
\hline Parameter & $\begin{array}{c}\text { Onset phase } \\
\text { (EAE vs control) }\end{array}$ & $\begin{array}{c}\text { Peak phase } \\
\text { (EAE vs control) }\end{array}$ & $\begin{array}{c}\text { Chronic phase } \\
\text { (EAE vs control) }\end{array}$ & $\begin{array}{c}\text { Onset } v s \\
\text { peak phase }\end{array}$ & $\begin{array}{c}\text { Peak } v s \\
\text { chronic phase }\end{array}$ & $\begin{array}{c}\text { Onset } v s \\
\text { chronic phase }\end{array}$ \\
\hline $\begin{array}{l}\text { Total leukocyte } \\
\text { density }\left[\text { area, } \mu \mathrm{m}^{2} \text { ] }\right.\end{array}$ & $\begin{array}{c}90654 \pm 28966 \\
v s 2474 \pm 790 \\
\mathrm{p}<0.0001\end{array}$ & $\begin{array}{c}124097 \pm 48195 \\
v s 1009 \pm 292 \\
\mathrm{p}=0.0012\end{array}$ & $\begin{array}{c}68546 \pm 23228 \\
v s 982 \pm 186 \\
\mathrm{p}=0.0013\end{array}$ & $\mathrm{p}=0.0046$ & $\mathrm{p}<0.0001$ & $\mathrm{p}=0.0037$ \\
\hline $\begin{array}{l}\text { T cells } \\
{\left[\text { cells } / \mathrm{mm}^{2}\right]}\end{array}$ & $\begin{array}{c}1149 \pm 340 \\
v s 14 \pm 5 \\
\mathrm{p}=0.0002\end{array}$ & $\begin{array}{c}2013 \pm 693 \\
v s 14 \pm 4 \\
\mathrm{p}=0.0001\end{array}$ & $\begin{array}{c}415 \pm 158 \\
v s 13 \pm 4 \\
\mathrm{p}<0.0001\end{array}$ & $\mathrm{p}<0.0001$ & $\mathrm{p}<0.0001$ & $\mathrm{p}<0.0001$ \\
\hline $\begin{array}{l}\text { Macrophage/microglia } \\
\left.\text { density [area, } \mu \mathrm{m}^{2}\right]\end{array}$ & $\begin{array}{c}87653 \pm 28563 \\
v s 5183 \pm 1901 \\
p<0.0001\end{array}$ & $\begin{array}{c}143202 \pm 37075 \\
\text { vs } 11828 \pm 2961 \\
\text { p }<0.0001\end{array}$ & $\begin{array}{c}123526 \pm 33754 \\
\text { vs } 11198 \pm 3837 \\
p<0.0001\end{array}$ & $\mathrm{p}<0.0001$ & $\mathrm{p}=0.1053$ & $\mathrm{p}=0.0009$ \\
\hline $\begin{array}{l}\text { Oligodendrocyte } \\
\text { density [area, } \mu \mathrm{m}^{2} \text { ] }\end{array}$ & $\begin{array}{c}121391 \pm 35603 v s \\
79879 \pm 9872 \\
p=0.024\end{array}$ & $\begin{array}{c}94413 \pm 29815 \\
\text { vs } 64925 \pm 19537 \\
\mathrm{p}=0.0039\end{array}$ & $\begin{array}{c}31532 \pm 11378 \\
\text { vs } 63479 \pm 13669 \\
\mathrm{p}=0.0002\end{array}$ & $\mathrm{p}=0.1037$ & $\mathrm{p}<0.0001$ & $\mathrm{p}<0.0001$ \\
\hline $\begin{array}{l}\text { Axonal damage } \\
\text { density }\left[\text { area, } \mu \mathrm{m}^{2}\right]\end{array}$ & $\begin{array}{l}2383 \pm 494 \\
\quad \text { vs } 0 \\
\mathrm{p}<0.0001\end{array}$ & $\begin{array}{c}4442 \pm 1483 \\
\text { vs } 0 \\
\mathrm{p}<0.0001\end{array}$ & $\begin{array}{c}3722 \pm 1253 \\
\text { vs } 0 \\
\mathrm{p}<0.0001\end{array}$ & $\mathrm{p}=0.0001$ & $\mathrm{p}=0.2062$ & $\mathrm{p}=0.0083$ \\
\hline $\begin{array}{l}\text { Surviving neurons } \\
{\left[\text { cells } / \mathrm{mm}^{2}\right]}\end{array}$ & $\begin{array}{c}1052 \pm 169 \\
\text { vs } 1109 \pm 171 \\
\mathrm{p}=0.8195\end{array}$ & $\begin{array}{c}1037 \pm 82 \\
v s 1063 \pm 205 \\
\mathrm{p}=0.5407\end{array}$ & $\begin{array}{c}805 \pm 156 \\
v s 1056 \pm 117 \\
\mathrm{p}<0.0001\end{array}$ & $\mathrm{p}=0.5254$ & $\mathrm{p}<0.0001$ & $\mathrm{p}<0.0001$ \\
\hline
\end{tabular}

Values represent mean \pm SEM, $n=6$ per group. Cells were identified by immunofluorescent staining as described in Methods.

coincided with initial neurological symptoms and remained stable in the further course of the disease [26]. However, as demonstrated in the latter study, myelin pathology (myelin swelling or loss) gradually accumulates with disease progression.

Axonal damage is not concomitant with neuronal death, since significant decrease in the number of surviving neurons was observed only in the chronic phase. Although the predominant view emphasizes the principal role of demyelination in the pathogenesis of MS/EAE, there is a growing evidence indicating the significance of neuronal loss in the disease [27]. Neuronal apoptosis in EAE can be induced by ER stress [28], matrix metalloproteinase 9 released by microglia [29] and perforins secreted by Tc cells [30]. We found that neuronal loss occurred relatively late, in the chronic phase of EAE. It suggests that neurons can survive demyelination and damage of their axons and that prolonged exposition to the inflammatory milieu is necessary to induce their death. This observation does not, however, agree with the results of a study of Vogt et al. [27] who reported death of neurons already in the early phase of an identical model of EAE and no further neuronal loss in the later phases. The discrepancy can stem from different quantification method employed be the latter authors (high precision stereology), and the time course of neuronal loss should be verified in further studies.

Histological correlates of autoimmune disease can provide important information concerning the underlying pathology. The analysis of cells involved and their behavior in the successive phases of EAE could also be useful in evaluating the therapeutic efficiency of anti-inflammatory, immunosuppressive and neuroprotective strategies employed in MS.

\section{Acknowledgements}

The authors thank Prof. Stefan Chłopicki from the Jagiellonian Centre for Experimental Therapeutics, Jagiellonian University, for providing laboratory facilities and Prof. Malgorzata Lekka from the Institute of Nuclear Physics, Krakow, for providing access to Cellsense Dimension Software. This study was supported by the statutory grant No K/ZDS/006248 from the Jagiellonian University Medical College to G.P-F.

\section{References}

1. Gold R, Linington C, Lassmann H. Understanding pathogenesis and therapy of multiple sclerosis via animal models: 70 years of merits and culprits in experimental autoimmune encephalomyelitis research. Brain. 2006; 129(Pt 8): 1953-1971, doi: 10.1093/brain/awl075, indexed in Pubmed: 16632554.

2. Stromnes IM, Goverman JM, Stromnes IM, et al. Active induction of experimental allergic encephalomyelitis. Nat Protoc. 2006; 1(4): 1810-1819, doi: 10.1038/nprot.2006.285, indexed in Pubmed: 17487163.

3. Storch MK, Stefferl A, Brehm U, et al. Autoimmunity to myelin oligodendrocyte glycoprotein in rats mimics the spectrum of multiple sclerosis pathology. Brain Pathol. 1998; 8(4): 681-694, indexed in Pubmed: 9804377.

4. Kuchroo VK, Anderson AC, Waldner H, et al. T cell response in experimental autoimmune encephalomyelitis (EAE): role 
of self and cross-reactive antigens in shaping, tuning, and regulating the autopathogenic $\mathrm{T}$ cell repertoire. Annu Rev Immunol. 2002; 20: 101-123, doi: 10.1146/annurev.immunol.20.081701.141316, indexed in Pubmed: 11861599.

5. Trapp BD, Nave KA. Multiple sclerosis: an immune or neurodegenerative disorder? Annu Rev Neurosci. 2008; 31: 247 -269, doi: 10.1146/annurev.neuro.30.051606.094313, indexed in Pubmed: 18558855.

6. Farooqi N, Gran B, Constantinescu CS. Are current disease-modifying therapeutics in multiple sclerosis justified on the basis of studies in experimental autoimmune encephalomyelitis? J Neurochem. 2010; 115(4): 829-844, doi: 10.1111/j.14714159.2010.06982.x, indexed in Pubmed: 20807309.

7. Hooke Laboratories, protocol EK-2110; available at: https:// //hookelabs.com/protocols/eaeAI_C57BL6.html.

8. Codarri L, Greter M, Becher B. Communication between pathogenic $\mathrm{T}$ cells and myeloid cells in neuroinflammatory disease. Trends Immunol. 2013; 34(3): 114-119, doi: 10.1016/j. it.2012.09.007, indexed in Pubmed: 23116549.

9. Rangachari M, Kuchroo VK. Using EAE to better understand principles of immune function and autoimmune pathology. J Autoimmun. 2013; 45: 31-39, doi: 10.1016/j.jaut.2013.06.008, indexed in Pubmed: 23849779.

10. Herrero-Herranz E, Pardo LA, Gold R, et al. Pattern of axonal injury in murine myelin oligodendrocyte glycoprotein induced experimental autoimmune encephalomyelitis: implications for multiple sclerosis. Neurobiol Dis. 2008; 30(2): 162-173, doi: 10.1016/j.nbd.2008.01.001, indexed in Pubmed: 18342527.

11. Krishnamoorthy G, Lassmann H, Wekerle H, et al. Spontaneous opticospinal encephalomyelitis in a double-transgenic mouse model of autoimmune $\mathrm{T}$ cell/B cell cooperation. J Clin Invest. 2006; 116(9): 2385-2392, doi: 10.1172/JCI28330, indexed in Pubmed: 16955140.

12. Pöllinger B, Krishnamoorthy G, Berer K, et al. Spontaneous relapsing-remitting EAE in the SJL/J mouse: MOG-reactive transgenic $\mathrm{T}$ cells recruit endogenous MOG-specific B cells. J Exp Med. 2009; 206(6): 1303-1316, doi: 10.1084/ /jem.20090299, indexed in Pubmed: 19487416.

13. Lisak RP, Nedelkoska L, Benjamins JA, et al. B cells from patients with multiple sclerosis induce cell death via apoptosis in neurons in vitro. J Neuroimmunol. 2017; 309: 88-99, doi: 10.1016/j.jneuroim.2017.05.004, indexed in Pubmed: 28601295.

14. Wu F, Cao W, Yang Y, et al. Extensive infiltration of neutrophils in the acute phase of experimental autoimmune encephalomyelitis in C57BL/6 mice. Histochem Cell Biol. 2010; 133(3): 313-322, doi: 10.1007/s00418-009-0673-2, indexed in Pubmed: 20063008.

15. Aubé B, Lévesque SA, Paré A, et al. Neutrophils mediate blood-spinal cord barrier disruption in demyelinating neuroinflammatory diseases. J Immunol. 2014; 193(5): 2438-2454, doi: 10.4049/jimmunol.1400401, indexed in Pubmed: 25049355.

16. Yi H, Guo C, Yu X, et al. Mouse CD11b+Gr-1+ myeloid cells can promote Th17 cell differentiation and experimental autoimmune encephalomyelitis. J Immunol. 2012; 189(9): 4295-4304, doi: 10.4049/jimmunol.1200086, indexed in $\mathrm{Pu}-$ bmed: 23034169.
17. Steinbach K, Piedavent M, Bauer S, et al. Neutrophils amplify autoimmune central nervous system infiltrates by maturing local APCs. J Immunol. 2013; 191(9): 4531-4539, doi: 10.4049/ /jimmunol.1202613, indexed in Pubmed: 24062488.

18. Ajami B, Bennett J, Krieger C, et al. Infiltrating monocytes trigger EAE progression, but do not contribute to the resident microglia pool. Nature Neuroscience. 2011; 14(9): 1142-1149, doi: 10.1038/nn.2887.

19. Liu C, Li Y, Yu J, et al. Targeting the shift from M1 to M2 macrophages in experimental autoimmune encephalomyelitis mice treated with fasudil. PLoS One. 2013; 8(2): e54841, doi: 10.1371/journal.pone.0054841, indexed in Pubmed: 23418431.

20. Chu F, Shi M, Lang Y, et al. The roles of macrophages and microglia in multiple sclerosis and experimental autoimmune encephalomyelitis. J Neuroimmunol. 2018; 318: 1-7, doi: 10.1016/j.jneuroim.2018.02.015, indexed in Pubmed: 29606295.

21. Shin T, Ahn M, Matsumoto Y. Mechanism of experimental autoimmune encephalomyelitis in Lewis rats: recent insights from macrophages. Anat Cell Biol. 2012; 45(3): 141-148, doi: 10.5115/acb.2012.45.3.141, indexed in Pubmed: 23094201.

22. Zawadzka M, Rivers LE, Fancy SPJ, et al. CNS-resident glial progenitor/stem cells produce Schwann cells as well as oligodendrocytes during repair of CNS demyelination. Cell Stem Cell. 2010; 6(6): 578-590, doi: 10.1016/j.stem.2010.04.002, indexed in Pubmed: 20569695.

23. Kotter MR, Li WW, Zhao C, et al. Myelin impairs CNS remyelination by inhibiting oligodendrocyte precursor cell differentiation. J Neurosci. 2006; 26(1): 328-332, doi: 10.1523/ /JNEUROSCI.2615-05.2006, indexed in Pubmed: 16399703.

24. Ozawa K, Suchanek G, Breitschopf H, et al. Patterns of oligodendroglia pathology in multiple sclerosis. Brain. 1994; 117(6): 1311-1322, doi: 10.1093/brain/117.6.1311.

25. Hisahara S, Okano H, Miura M. Caspase-mediated oligodendrocyte cell death in the pathogenesis of autoimmune demyelination. Neurosci Res. 2003; 46(4): 387-397, indexed in Pubmed: 12871760.

26. Recks MS, Stormanns ER, Bader J, et al. Early axonal damage and progressive myelin pathology define the kinetics of CNS histopathology in a mouse model of multiple sclerosis. Clin Immunol. 2013; 149(1): 32-45, doi: 10.1016/j.clim.2013.06.004, indexed in Pubmed: 23899992.

27. Vogt J, Paul F, Aktas O, et al. Lower motor neuron loss in multiple sclerosis and experimental autoimmune encephalomyelitis. Ann Neurol. 2009; 66(3): 310-322, doi: 10.1002/ /ana.21719, indexed in Pubmed: 19798635.

28. Cunnea P, Mháille ANí, McQuaid S, et al. Expression profiles of endoplasmic reticulum stress-related molecules in demyelinating lesions and multiple sclerosis. Mult Scler. 2011; 17(7): 808-818, doi: 10.1177/1352458511399114, indexed in Pubmed: 21382862.

29. Kauppinen TM, Swanson RA. Poly(ADP-ribose) polymerase-1 promotes microglial activation, proliferation, and matrix metalloproteinase-9-mediated neuron death. J Immunol. 2005; 174(4): 2288-2296, indexed in Pubmed: 15699164.

30. Zhao D, Feng F, Zhao C, et al. Role of perforin secretion from CD8+ T-cells in neuronal cytotoxicity in multiple sclerosis. Neurol Res. 2018; 40(1): 62-67, doi: 10.1080/01616412.2017.1398371, indexed in Pubmed: 29105578.

Submitted: 5 June, 2018

Accepted after reviews: $30 \mathrm{July}, 2018$ Available as AoP: 30 August, 2018 